OPEN ACCESS

Edited by:

Firas H. Kobeissy,

University of Florida,

United States

Reviewed by:

Corina Bondi,

University of Pittsburgh,

United States

Karim A. Sarhane,

University of Toledo, United States

Wael M. Y. Mohamed,

International Islamic University

Malaysia, Malaysia

*Correspondence:

Fei Liu

doctorlf@126.com

Specialty section:

This article was submitted

to Neurotrauma,

a section of the journal

Frontiers in Neurology

Received: 23 July 2017 Accepted: 09 February 2018 Published: 26 February 2018

Citation:

Xiao Y, Li G, Chen Y, Zuo Y, Rashid K, He T, Feng $H$, Zhang JH and Liu $F$ (2018) Milk Fat Globule-Epidermal

Growth Factor-8 Pretreatment

Attenuates Apoptosis and

Inflammation via the Integrin- $\beta 3$

Pathway after Surgical Brain

Injury in Rats.

Front. Neurol. 9:96.

doi: 10.3389/fneur.2018.00096

\section{Milk Fat Globule-Epidermal Growth Factor-8 Pretreatment Attenuates Apoptosis and Inflammation via the Integrin-\$3 Pathway after Surgical Brain Injury in Rats}

\author{
Yicai Xiao', Gaofeng Li², Yujie Chen³, Yuchun Zuo', Kauthar Rashid', Tibiao He', \\ Hua Feng ${ }^{3}$, John H. Zhang ${ }^{4}$ and Fei Liu ${ }^{1 *}$
}

'Department of Neurosurgery, The Third Xiangya Hospital, Central South University, Hunan, Changsha, China, ${ }^{2}$ Departments of Oncology, Zhuzhou Central Hospital, Hunan, Zhuzhou, China, ${ }^{3}$ Department of Neurosurgery, Southwest Hospital, Third Military Medical University, Chongqing, China, ${ }^{4}$ Neuroscience Research Center, Loma Linda University, Loma Linda, CA, United States

latrogenic brain injury inevitably occurs in neurosurgical operations, leading to brain edema, ischemia, intracranial hematoma, and other postoperative complications, eventually worsening neurological outcomes of patients. If apoptotic cells are not rapidly eliminated by phagocytic engulfment, they may communicate with surrounding cells to undergo secondary necrosis and releasing toxic signals. Recent studies have shown that milk fat globule-epidermal growth factor-8 (MFGE8), which promotes phagocytosis and inhibits inflammation, is an endogenous protective factor in response to brain infarction, Alzheimer's disease, subarachnoid hemorrhage, and prion disease. In the present study, we sought to investigate the different effects of both pretreated and posttreated recombinant milk fat globule-epidermal growth factor-8 (rhMFGE8) for the surgical brain injury (SBI) rat model and potential involvement of its receptor integrin $\beta 3$ for apoptosis and neuroinflammation after SBI. One hundred and sixty-seven male rats were employed in the preset study. Experiment 1 was performed to evaluate neurological scores and MFGE8, cleaved caspase-3 (CC3), and interleukine-1 beta (IL-1 $\beta$ ) levels at 3, 24, and $120 \mathrm{~h}$ after SBI. Experiment 2 was performed to evaluate the effects of rhMFGE8 pretreatment (10 min before SBI) and rhMFGE8 posttreatment ( $6 \mathrm{~h}$ after SBI) on brain edema at 24 and $72 \mathrm{~h}$ after SBI. Experiment 3 was performed to evaluate the potential anti-apoptotic and anti-inflammatory effects of rhMFGE8 pretreatment and posttreatment. Experiment 4 sought to investigate the involvement of the integrin- $\beta 3$ signal in the effects of MFGE8 pretreatment. Our data showed rhMFGE8 pretreatment alleviated neurological deficits and decreased brain water content and apoptotic cells in the SBI model, which exhibited neurological dysfunction, apoptosis, and inflammation. Meanwhile, MFGE8 siRNA, which inhibited endogenous MFGE8 expression, significantly increased IL-1 $\beta$, TUNEL positive cells, and CC3. Furthermore, knockdown of its receptor integrin $\beta 3$ by siRNA abolished the effects of rhMFGE8 in the SBI model. In conclusion, we found that 
rhMFGE8 pretreatment effectively alleviated neurological deficits and decreased brain water content and apoptotic cells in the SBI model through the MFGE8/integrin- $\beta 3$ pathway, and treatment time was an important factor in achieving curative effects. Therefore, MFGE8 pretreatment may serve as a promising therapeutic strategy for SBI patients.

Keywords: milk fat globule-epidermal growth factor-8, surgical brain injury, apoptosis, inflammation, integrin $\beta 3$

\section{INTRODUCTION}

Iatrogenic brain injury inevitably occurs in neurosurgical operations, leading to brain edema, ischemia, intracranial hematoma, and other postoperative complications, eventually worsening neurological outcomes of patients (1). The mechanisms of this damage include cortical incision, electrocauterization, retraction, and vascular or microvascular occlusion. A surgical brain injury (SBI) rodent model has been developed and demonstrated brain edema, cell apoptosis, inflammation, and oxidative stress in the peri-resection area (2-6). Among these pathophysiologies, inflammation plays a pivotal role in the secondary brain injuries due to different etiologies, including but not limited to trauma, ischemia, neurodegeneration, and excitotoxicity (7-9). If apoptotic cells are not rapidly eliminated by phagocytic engulfment, they may communicate with surrounding cells to undergo secondary necrosis and releasing toxic signals, such as the inflammatory factor interleukine-1 beta (IL-1 $\beta$ ) (10-12). Therefore, timely apoptotic cell clearance could alleviate inflammatory damage.

Milk fat globule-epidermal growth factor-8 (MFGE8, also called lactadherin), a multifunctional glycoprotein which was originally understood as part of the milk fat globule membrane (13-15), is instrumental in cell-cell interactions and diverse physiological and pathophysiological functions, including fertilization, angiogenesis, and phagocytosis of apoptotic cells (16). Recent studies have shown that MFGE8, which promotes phagocytosis and inhibits inflammation, is an endogenous protective factor in response to brain infarction $(17,18)$, Alzheimer's disease $(19,20)$, subarachnoid hemorrhage (SAH) (16), and prion disease (21). However, Kinugawa et al. reported that MFGE8 does not orchestrate the apoptotic neurons clearance in a Parkinson's disease mouse model (22). Moreover, Neher et al. found that MFGE8 $8^{-/}$mice had therapeutic targets even with a lack of MFGE8 expression in transient cerebral ischemia (23). MFGE8 thus has dual effects in different models.

To date, the effects of MFGE8 in the SBI model are unclear. Moreover, SBI patients show similarities to the SBI model after pretreatment with drugs, in contrast to stroke and trauma patients. Therefore, in the present study, we sought to investigate the different effects of both pretreated- and posttreated recombinant milk fat globule-epidermal growth factor-8 (rhMFGE8) for SBI rat model and potential involvement of its receptor integrin $\beta 3$ for apoptosis and neuroinflammation after SBI. The results preliminarily demonstrated that MFGE8 is a promising therapeutic target for SBI and that treatment time was an important factor in achieving a curative effect.

\section{MATERIALS AND METHODS}

\section{Experimental Design}

One hundred and sixty-eight male Sprague-Dawley rats, age between 8 and 12 weeks, weighing 280-350 g, were employed in the present study. Rats were housed in a humidity and temperature-controlled room with food and water ad libitum. The light was controlled in a 12 -h light/dark cycle. These rats were acclimatized for more than 3 days before surgical procedures. All experimental protocols were approved by the Ethics Committee of Central South University and performed according to the eighth edition of the National Institutes of Health Guide for the Care and Use of Laboratory Animals and reported according to the ARRIVE guideline.

Experiments designs are illustrated in Figure 1. Experiment 1 (Figure 1A) was performed to evaluate neurological scores $(n=6$ at each timepoint) and MFGE8, cleaved caspase-3 (CC3), and

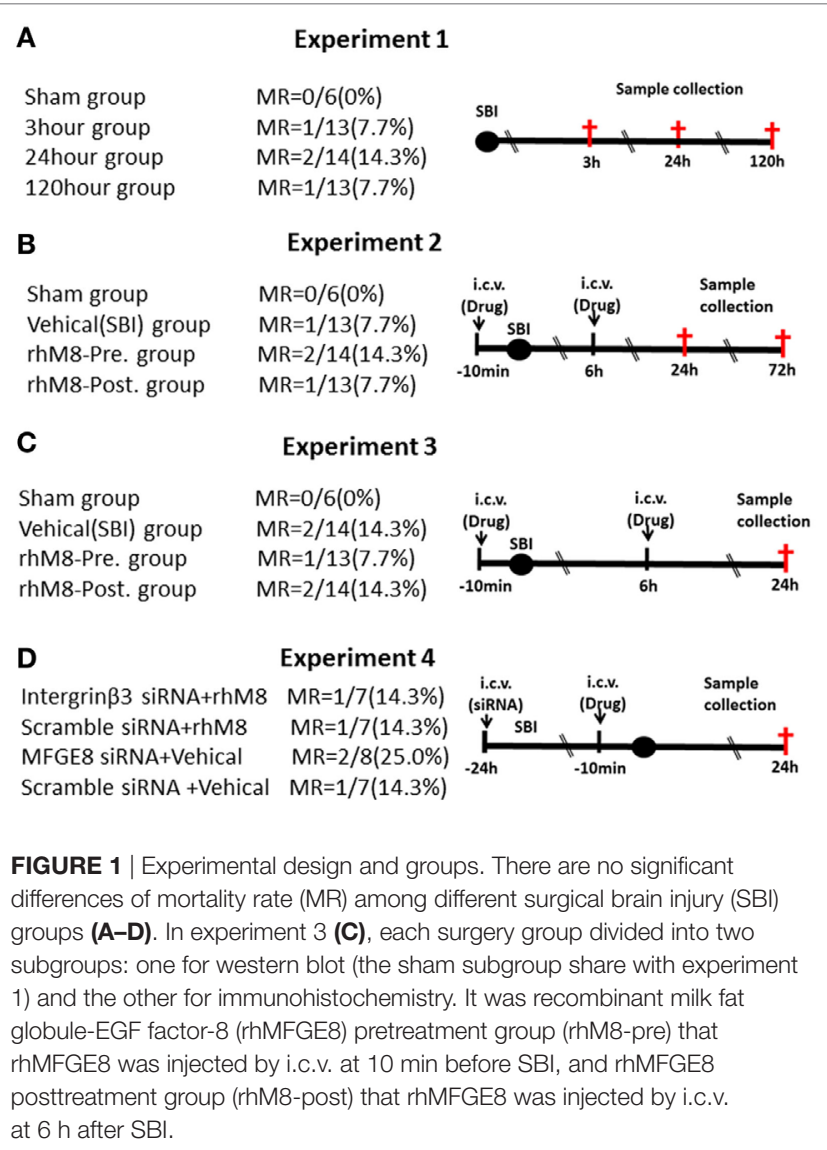


IL- $1 \beta$ levels ( $n=6$ at each timepoint) at 3, 24, and $120 \mathrm{~h}$ after SBI. Experiment 2 (Figure 1B) was performed to evaluate the effects of rhMFGE8 pretreatment (10 min before SBI) and rhMFGE8 posttreatment ( $6 \mathrm{~h}$ after SBI) on brain edema ( $n=6$ at each timepoint) at 24 and $72 \mathrm{~h}$ after SBI. Experiment 3 (Figure 1C) was performed to evaluate the potential anti-apoptotic and anti-inflammatory effects of rhMFGE8 pretreatment and posttreatment $(n=6)$. Experiment 4 (Figure 1D) sought to investigate the involvement of the integrin- $\beta 3$ signal in the effects of MFGE8 pretreatment ( $n=6$ for each group).

\section{Surgical Brain Injury}

As previously described (6), the SBI model was performed by partially resecting the right frontal lobe of rat brain. Briefly, rats were intraperitoneally anesthetized with $50-\mathrm{mg} / \mathrm{kg}$ sodium pentobarbital, followed with $0.5-\mathrm{mg} / \mathrm{kg}$ atropine intraperitoneally to inhibit respiratory secretions. Under a surgical dissecting microscope, a midline incision was made in scalp, and a 4-mm edge square cranial window, beginning at the bregma at right frontal skull, was removed using a bone drill. Then, the dura was excised. A partial right frontal lobectomy was made through two separate incisions leading away from the bregma along the sagittal and coronal, approximately 2-mm lateral to the sagittal suture and 1-mm proximal to the coronal suture. Once the brain tissue was excised, electrocautery was applied briefly (1-2 s total) to the cut surfaces used for hemostasis. Sham animals underwent only a craniotomy.

\section{Intracerebroventricular Infusion}

Intracerebroventricular infusion was performed as previously described (24). Briefly, rats were placed in a stereotactic apparatus after they were anesthetized with sodium pentobarbital. Then, the skin was incised, and a burr hole was drilled at the following coordinates relative to the bregma: $1.5-\mathrm{mm}$ posterior and $1.0-\mathrm{mm}$ lateral (left). The needle of a 10- $\mu \mathrm{L}$ Hamilton syringe (Microliter 701; Hamilton Company, Reno, NV, USA) was inserted below the horizontal plane of the skull. Drugs, which included rhMFGE8 (Sigma, $3.3 \mu \mathrm{g} / 3 \mu \mathrm{L}$ ), MFGE8 siRNA, integrin- $\beta 3$ siRNA, or scrambled siRNA (500 pmol/3 $\mu \mathrm{L}$, Santa Cruz Biotechnology), were injected at a rate of $0.5 \mu \mathrm{L} / \mathrm{min}$ by a pump on schedule according to the design.

\section{Neurobehavioral Test}

Neurobehavioral scores included modified Garcia scoring test and beam balance test at $1 \mathrm{~h}$ before euthanasia (25). Tests were performed by a blinded observer. The modified Garcia test score ranges from 0 to 18 , which was assigned based on spontaneous activity, spontaneous movement of all limbs, fore paw outstretching, climbing, body proprioception, and response to whisker stimulation. The beam balance test (0-4) was based on the walking distance on a $45-\mathrm{cm}$ long and $22.5-\mathrm{mm}$ diameter wooden beam between two platforms. Briefly, rats were placed perpendicularly on the midpoint of the beam and allowed to traverse the beam for $60 \mathrm{s.} 0$ point for "Does not move and falls off the rod within $30 \mathrm{~s}$," 1 point for "Does not move but stays on the rod for more than 30 s (in any manner)," 2 point for "Moves less than half the distance to the platform $(22.5 \mathrm{~cm})$ in $\leq 60 \mathrm{~s}$," 3 point for "Moves at least half the distance to the platform $(22.5 \mathrm{~cm})$ in $\leq 60 \mathrm{~s}$," and 4 point for "Reaches the platform or moves at least $45 \mathrm{~cm}$ in $\leq 60 \mathrm{~s}$."

\section{Brain Water Content}

Rat brains were removed under anesthesia at 24 or $72 \mathrm{~h}$ after surgery. Brains were quickly separated into six parts: right/left frontal, right/parietal, cerebellum, and brain stem. The coronal middle line was defined as the cut-off for the frontal lobe and parietal lobe. Samples were immediately weighed on a high-precision balance (wet weight) and after drying in an oven at $105^{\circ} \mathrm{C}$ at $48 \mathrm{~h}$ later (dry weight). The percentage of brain water content was calculated as (wet weight-dry weight)/wet weight $\times 100 \%$.

\section{Western Blot Analysis}

The right frontal tissues were collected $24 \mathrm{~h}$ after SBI. Samples were snap-frozen in liquid nitrogen and stored at $80^{\circ} \mathrm{C}$ until use. Western blot was performed as described previously (25). Primary antibodies against MFGE8, $\beta$-Actin (1:4,000, Santa Cruz Biotechnology, Santa Cruz, CA, USA), IL-1 $\beta$ (1:2,000, Abcam, Cambridge, MA, USA), and CC3 (1:2,000, Cell Signaling Technology, Danvers, MA, USA) were used.

\section{Immunohistochemistry}

Animals were euthanized at $24 \mathrm{~h}$ under deep anesthesia. Brain tissue was fixed with sucrose and formalin. Then, $10-\mu \mathrm{m}$ coronal sections were cut in a cryostat. As described previously (24), immunofluorescence staining was performed by using an in situ cell death detection kit (Roche, Indianapolis, IN, USA) as the manufacturer's instruction. Four views/pictures were taken along the periphery of surgical resection and calculated for each rat. The TUNEL-positive cells were quantified at $\times 200$ magnification in a blinded manner by other researchers and expressed as cells $/ \mathrm{mm}^{2}$.

\section{Statistical Analysis}

Statistical analysis was performed using GraphPad Prism 5 (GraphPad Software Inc., San Diego, CA, USA). Quantitative data were presented as the mean \pm SEM. One-way analysis of variance (ANOVA) followed by Tukey's multiple comparisons test was used for different groups' comparison. Chi-square tests were used for behavior score analyses. $P<0.05$ was considered statistically significant.

\section{RESULTS}

All sham-operated rats survived. A total of 168 male rats underwent SBI; 18 rats died after SBI, and the mortality of SBI rats was $10.7 \%$. But the mortality did not exhibit significantly different among the experimental groups (Figure 1, $P>0.05$ ).

\section{MFGE8, CC3, and IL-1 $\beta$ Expression Increased after SBI}

At $3 \mathrm{~h}$ after SBI, the rat models exhibited obvious neurological deficits in the modified Garcia score (Figure 2A, $P<0.05$ ) and beam balance score (Figures $\mathbf{2 B}, P<0.05$ ) comparing to sham rats, and these differences further increased at $24 \mathrm{~h}$ after SBI $(P<0.05)$. However, compared with sham rats, the SBI rats 
A

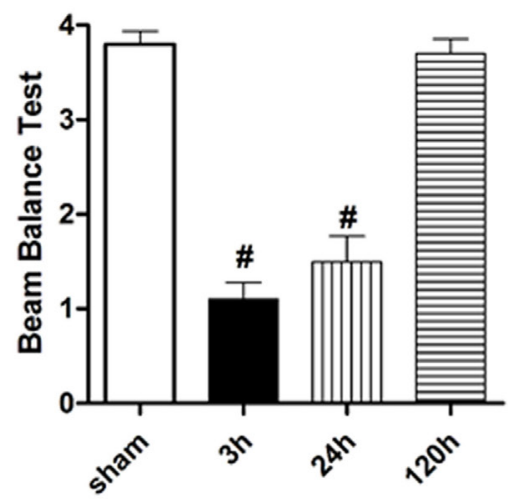

C

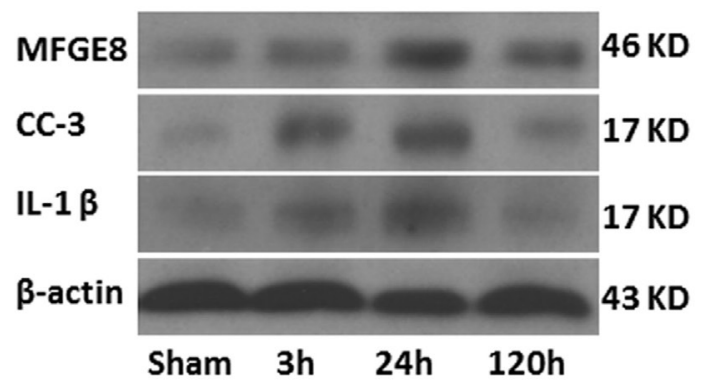

E

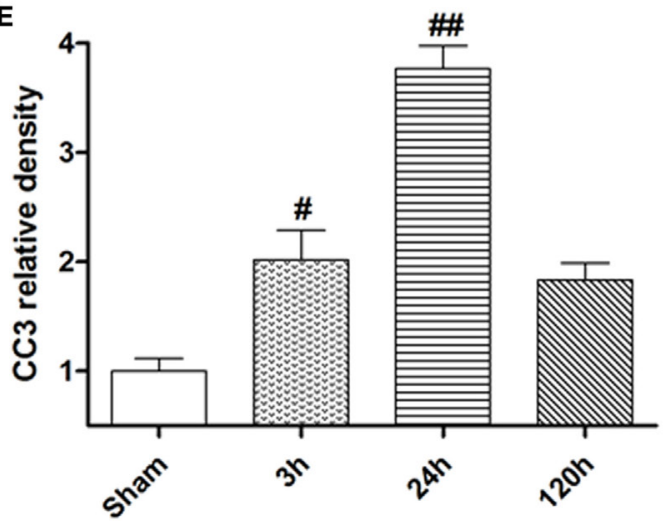

B
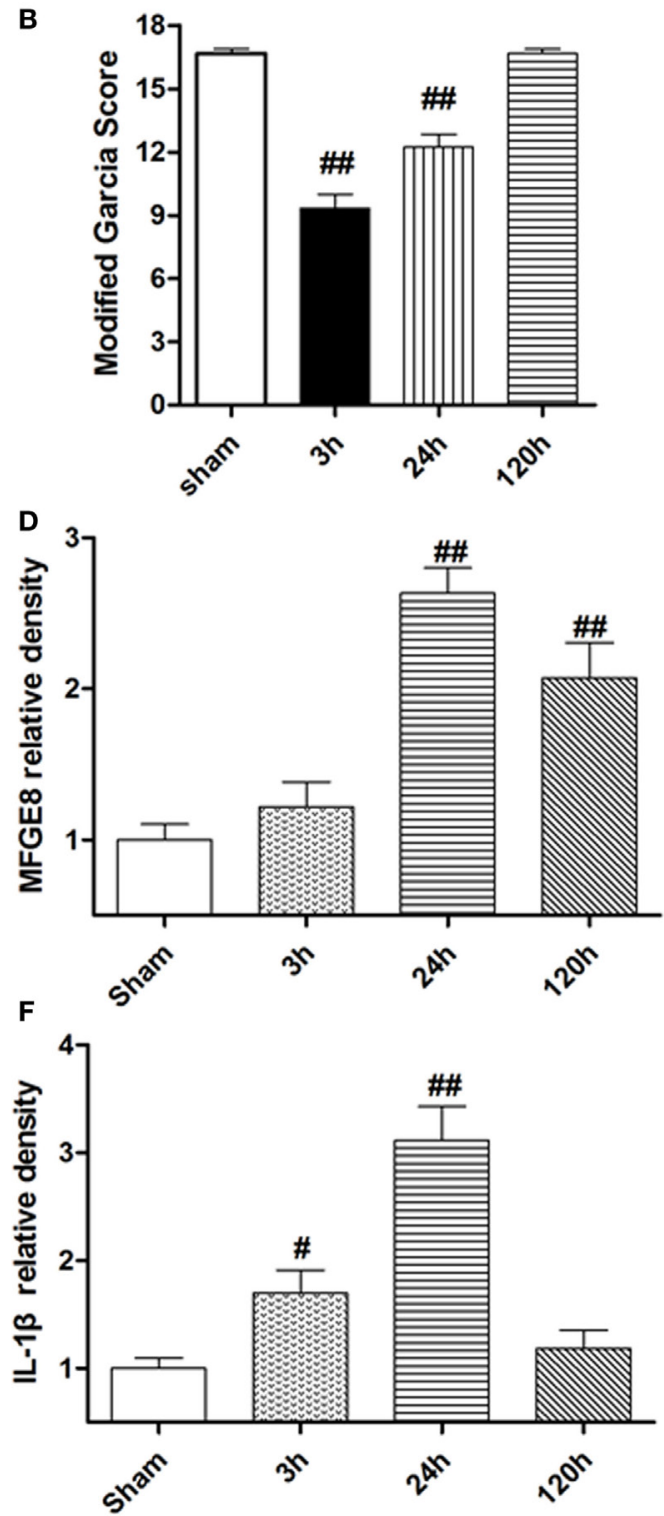

FIGURE 2 | Time course of neurological scores, and expression of milk fat globule-EGF factor-8 (MFGE8), cleaved caspase-3 (CC3), and interleukine-1 beta (IL-1 $\beta$ ) after surgical brain injury (SBI). (A,B) Animals showed severely neurological deficits from $3 \mathrm{~h}$ after subarachnoid hemorrhage, improved as time passed (at $120 \mathrm{~h}$ after SBI), and was not significant difference with sham. Representative blots (C) and quantitative analysis of MFGE8, CC3, and IL-1 $\beta$ (D-F) show the levels of MFGE8, CC3, and IL-1 $\beta$ all increased after SBI and peaked at $24 \mathrm{~h}$, but expression of MFGE8 increasing (D) was not significant at $3 \mathrm{~h}$ after SBI. $n=6$ for each time point. ${ }^{\#} P<0.05$ vs. sham group and ${ }^{\# \# P}<0.01$ vs. sham group.

showed no significant differences in neurological function on the modified Garcia score and beam balance score at $120 \mathrm{~h}$ after SBI (Figures 2A,B, $P>0.05$ ). Western blots demonstrated that MFGE8, CC3, and IL-1 $\beta$ were all poorly expressed in sham rats (Figures 2C-F). They all increased from $3 \mathrm{~h}$ after SBI, with a peak at $24 \mathrm{~h}$, which lasted $120 \mathrm{~h}$ after SBI (Figures $2 \mathrm{C}-\mathrm{F}, P<0.01$ ). The increase in CC3 and IL-1 $\beta$ was significant at $3 \mathrm{~h}$ after SBI compared with that of sham rats (Figures $2 \mathrm{E}, \mathrm{F}, P<0.05$ ), but MFGE8 expression was not significantly altered (Figure 2D, $P>0.05)$. However, MFGE8 was still significantly higher than that in the sham group at $120 \mathrm{~h}$ after SBI $(P<0.01)$, but CC3 and IL-1 $\beta$ showed no differences compared with the expression in sham rats (Figures 2C-F, $P>0.05$ ).

\section{Administration of rhMFGE8 Alleviated Neurological Deficits and Decreased Brain Water Content in SBI Rats}

There was a significant decrease in the modified Garcia score (Figures 3A,C $P<0.01$ ) and the beam balance score (Figures 3B,D, $P<0.05$ ) and aggravated brain water content in the right frontal lobe (ipsilateral) (Figures 4 A,B, $P<0.01$ ) in all 

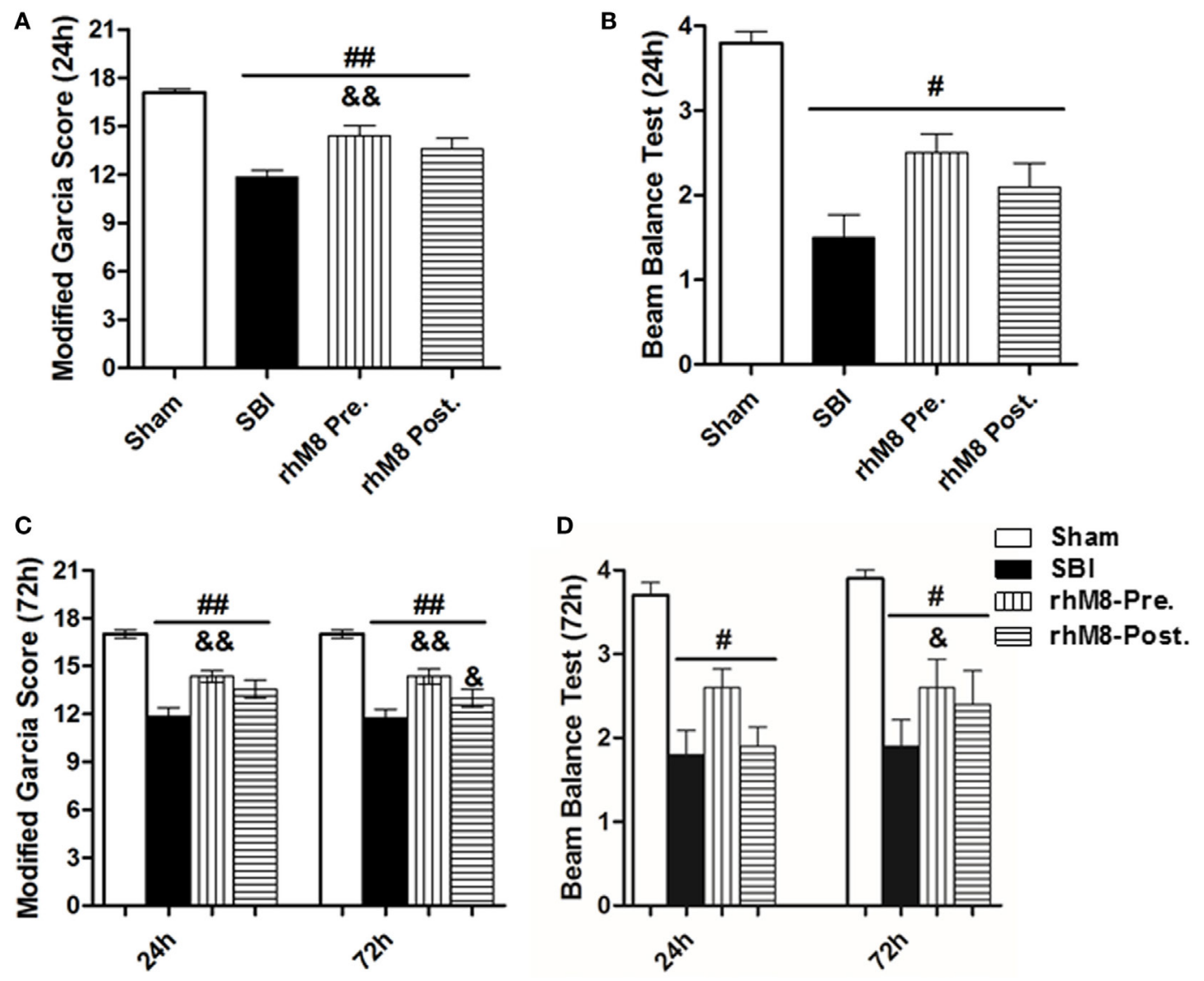

FIGURE 3 | The different effect between recombinant milk fat globule-EGF factor-8 (rhMFGE8) pretreatment (rhM8-pre) and rhMFGE8 posttreatment (rhM8-post) on neurological scores at 24 and $72 \mathrm{~h}$ after surgical brain injury (SBI). (A,B) rhM8-pre significantly improved the modified Garcia neurological function at $24 \mathrm{~h}$. However, rhM8-post was not significant. (C,D) At 72 h, rhM8-pre significantly improved the modified Garcia neurological function and balance function, but rhM8-pre only significantly improved the modified Garcia neurological function. $n=6$ for each group ${ }^{\sharp} P<0.05$ vs. sham group, ${ }^{\# \#} P<0.01$ vs. sham group, ${ }^{8} P<0.05$ vs. SBI group, and ${ }^{\&} \mathrm{P} P<0.01 \mathrm{vs.} \mathrm{SBl}$ group.

rats subjected to SBI, with or without treatment, at 24 and $72 \mathrm{~h}$ compared with rats in the sham group.

Recombinant milk fat globule-epidermal growth factor-8 was administered intracerebroventricularly at two time points: 10 min before SBI (rhMFGE8 pretreatment) and $6 \mathrm{~h}$ after SBI (rhMFGE8 posttreatment). rhMFGE8 pretreatment significantly improved the modified Garcia score at $24 \mathrm{~h}$ (Figure 3A, $P<0.01$ ) and $72 \mathrm{~h}$ (Figure 3C, $P<0.05$ ) and increased the beam balance score only at $72 \mathrm{~h}$ (Figure 3D, $P<0.05$ ). Moreover, rhMFGE8 pretreatment significantly decreased the brain water content at $24 \mathrm{~h}$ (Figure 4A, $P<0.05$ ) and $72 \mathrm{~h}$ (Figure 4B, $P<0.01$ ). However, posttreatment only increased the modified Garcia score (Figure 3C, $P<0.05$ ) and decreased the brain water content at $72 \mathrm{~h}$ (Figure 4B, $P<0.01$ ).

\section{Administration of rhMFGE8 Decreased TUNEL-Positive Cells $24 \mathrm{~h}$ after SBI, and Pretreatment Was Effective}

TUNEL staining in the right frontal lobe indicated that the sham group had few TUNEL-positive cells (Figure 5). Stained cells increased substantially along the periphery of surgical resection in the SBI model. Administration of rhMFGE8 decreased the abundance of positive cells (Figure 5A), but only pretreatment had significant effects (Figures 5B,C, $P<0.01)$.

\section{Administration of rhMFGE8 Decreased Expression of $\mathrm{CC} 3$ and IL-1 $\beta$ at $24 \mathrm{~h}$ after SBI, and Pretreatment Was More Effective than Posttreatment}

The treatments all significantly increased the protein levels of MFGE8 (Figures 6A,B, $P<0.01$ ), CC3 (Figures 6A,C, $P<0.01$ ), and IL-1 $\beta$ (Figures $\mathbf{6 A}, \mathbf{D}, P<0.01$ ) in the SBI model. Administration of rhMFGE8 further increased the protein level of MFGE8 (Figures 6A-C, $P<0.01$ ). Pretreatment significantly decreased the expression of CC3 (Figures 6A,C, $P<0.05$ ) and IL-1 $\beta$ (Figures 6A,D, $P<0.01$ ). However, posttreatment, which only significantly decreased the expression of IL- $1 \beta$ (Figures 6A,D, $P<0.05$ ), did not significantly decrease CC3 expression (Figures 6A,C, $P>0.05$ ). 

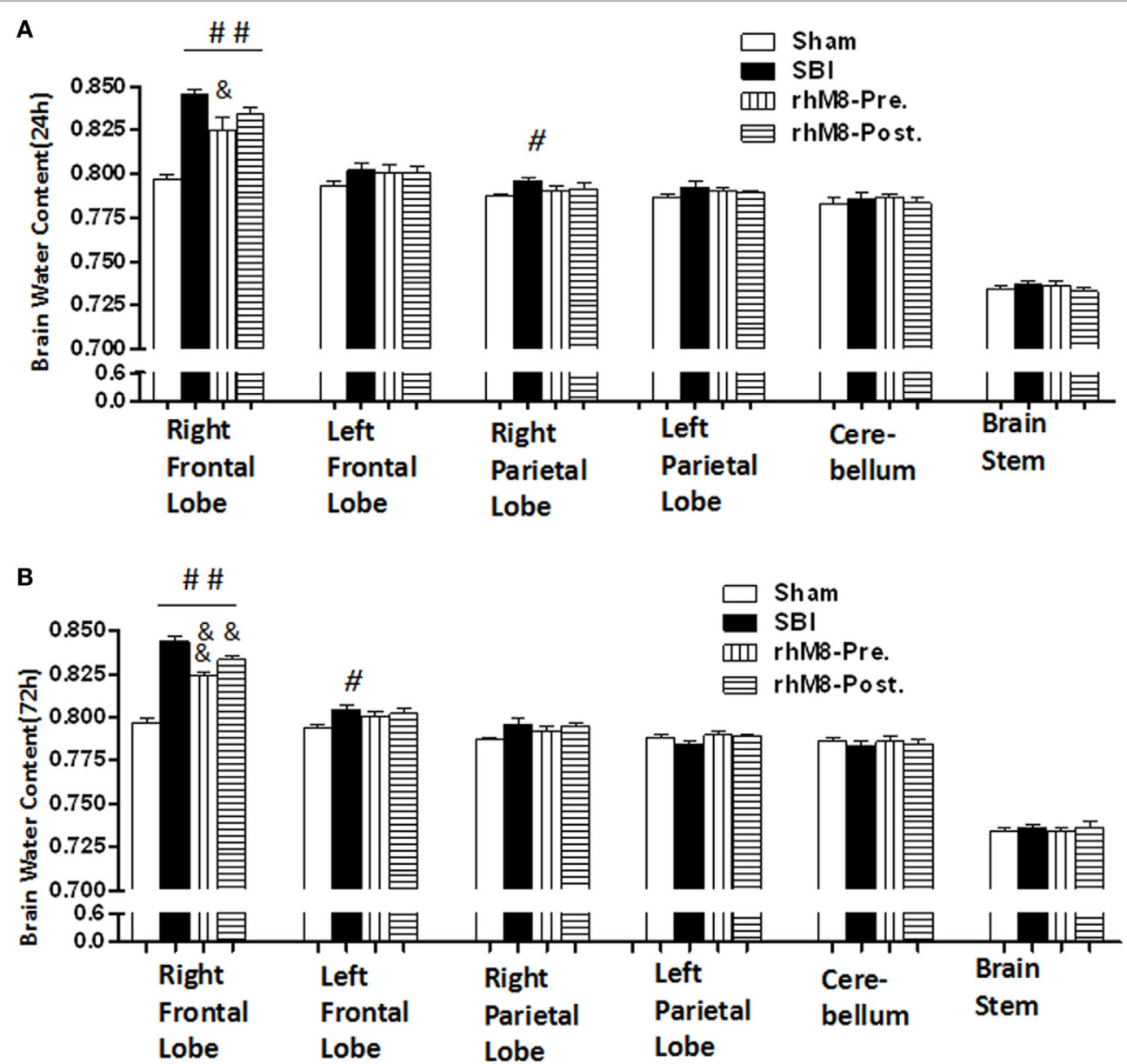

FIGURE 4 | The different effects between recombinant milk fat globule-EGF factor-8 (rhMFGE8) pretreatment (rhM8-pre) and rhMFGE8 posttreatment (rhM8-post) on brain edema at 24 and $72 \mathrm{~h}$. (A,B) rhM8-pre significantly reduced brain water content in right (ipsilateral) frontal lobes at 24 and $72 \mathrm{~h}$. However, the decreasing of brain water content in rhM8-post was not significant. $n=6$ for each group. ${ }^{*} P<0.05$ vs. sham group, ${ }^{\#} P<0.01$ vs. sham group, ${ }^{\&} P<0.05$ vs. surgical brain injury (SBI) group, and \&\&P $<0.01$ vs. SBI group.

\section{Knockdown of Endogenous MFGE8 Increased the Expression of $\mathrm{CC} 3$} and IL-1 $\beta$ at $24 \mathrm{~h}$ after SBI

Milk fat globule-epidermal growth factor-8 siRNA pretreatment significantly decreased the modified Garcia score at $24 \mathrm{~h}$ after SBI (Figure 7A, $P<0.05$ ) and knocked down endogenous MFGE8 protein expression (Figures $\mathbf{7 B}, \mathbf{C}, P<0.05$ ), which increased CC6 (Figures 7B,E, $P<0.05$ ) and IL-1 $\beta$ (Figures 7B,F, $P<0.05$ ) expression. In contrast, scramble siRNA had no significant effects $(P>0.05)$.

\section{Knockdown of Integrin- $\beta 3$ Receptor Abolished the Neuroprotective Effects of rhMFGE8 Treatment at $24 \mathrm{~h}$ after SBI}

Integrin- $\beta 3$ siRNA blocked the rhMFGE8-mediated increase in Garcia score at $24 \mathrm{~h}$ after SBI $(P<0.05)$. Integrin- $\beta 3$ siRNA efficiently inhibited integrin- $\beta 3$ protein expression (Figures $7 \mathbf{B}, \mathbf{C}$, $P<0.05$ ), which has no effects on the protein levels of MFGE8
(Figures 7B,D, $P>0.05$ ). Furthermore, integrin- $\beta 3$ siRNA abolished the rhMFGE8-mediated decrease in expressions of CC3 (Figures 7B,E, $P<0.05$ ) and IL-1 $\beta$ (Figures 7B,E, $P<0.05$ ) at $24 \mathrm{~h}$ after SBI.

\section{DISCUSSION}

In the present study, we investigated the different effects of rhMFGE8 pretreatment and posttreatment and whether the MFGE8/ integrin- $\beta 3$ pathway was involved in the anti-apoptotic and anti-inflammatory effects in a rat model of SBI. Our data showed that rhMFGE8 pretreatment alleviated neurological deficits and decreased brain water content and apoptotic cells in the SBI model, which exhibited neurological dysfunction, apoptosis, and inflammation. Meanwhile, MFGE8 siRNA, which inhibited endogenous MFGE8 expression, significantly increased apoptotic cells and the protein levels of CC3 and IL-1 $\beta$. Furthermore, knockdown of its receptor integrin $\beta 3$ by siRNA abolished the effects of rhMFGE8 in the SBI model. 


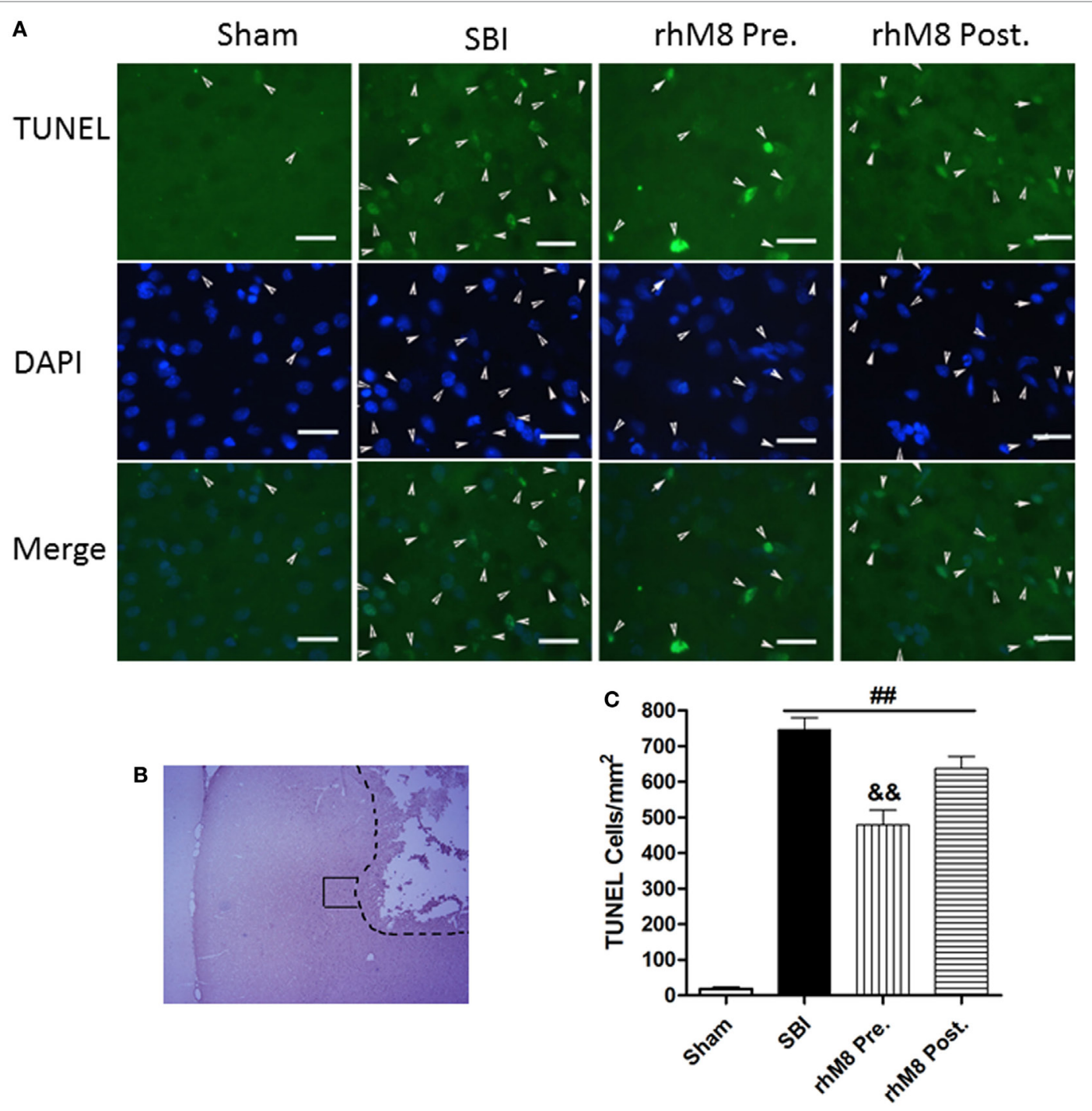

FIGURE 5 | The different effects between recombinant milk fat globule-EGF factor-8 (rhMFGE8) pretreatment (rhM8-pre) and rhMFGE8 posttreatment (rhM8-post) on TUNEL-positive cells at $24 \mathrm{~h}$ after surgical brain injury (SBI). (A) Representative microphotographs showed the colocalization of TUNEL (green)-positive with DAPI (blue) cells along the periphery of surgical resection (B) at $24 \mathrm{~h}$ after SBI. (C) Semi-quantitative analysis of TUNEL-positive cells showed that the number of apoptotic cells was significantly increased after SBI and rhMFGE8 significantly decreased the number of apoptotic cells, but rhM8-pre had better effective. Scale bar = 50 $\mu$ m; $n=6$ for each group. ${ }^{\#} P<0.01$ vs. sham group, ${ }^{\&} P<0.05$ vs. SBI group, and ${ }^{\&} P<0.01$ vs. SBI group.

Milk fat globule-epidermal growth factor-8 is a secreted protein for the regulation of apoptotic cells clearance which contains two characteristic functional domains: EGF-like domains, including a RGD-containing sequence that binds to integrin of phagocytic cells, and a blood coagulation factor V/VIII segment binding to phosphatidylserine, which is usually located at inner leaflet of plasma membrane and only exposed in the process of apoptosis $(11,13-15,26)$. Therefore, the full engulfment process and apoptotic cells clearance cannot be completed without MFGE8 (27). In the present study, we showed that there were many apoptotic cells surrounding the corticotomy region and increased CC3 protein in the right frontal lobe at $24 \mathrm{~h}$ after SBI, which was consistent with previous studies of the SBI model (4). Following administration of rhMFGE8, TUNEL-positive cells were decreased at the periphery of surgical resection, and the CC3 protein level decreased. MFGE8 siRNA inhibited endogenous MFGE8 expression, which increased the expression of CC3 and IL- $1 \beta$ at $24 \mathrm{~h}$ after SBI. These results were consistent with previous studies showing that MFGE8 promoted phagocytosis of microglia in an SAH model and that MFGE8 deficiency is detrimental (16). For the engulfment of apoptotic bodies, a specific vitronectin receptor (integrin $\alpha v \beta 3$ or integrin $\alpha v \beta 5$ ), which is expressed on phagocytes, binds MFGE8opsonized apoptotic cells $(28,29)$. In the present study, knockdown of integrin $\beta 3$ by siRNA abolished the anti-inflammatory and anti-apoptotic effects of rhMFGE8 in the SBI model, similar to observations made in $\mathrm{SAH}(16,24)$. These findings indicate that rhMFGE8 enhances apoptotic cells engulfment through the MFGE8/integrin- $\beta 3$ pathway in the SBI model. 

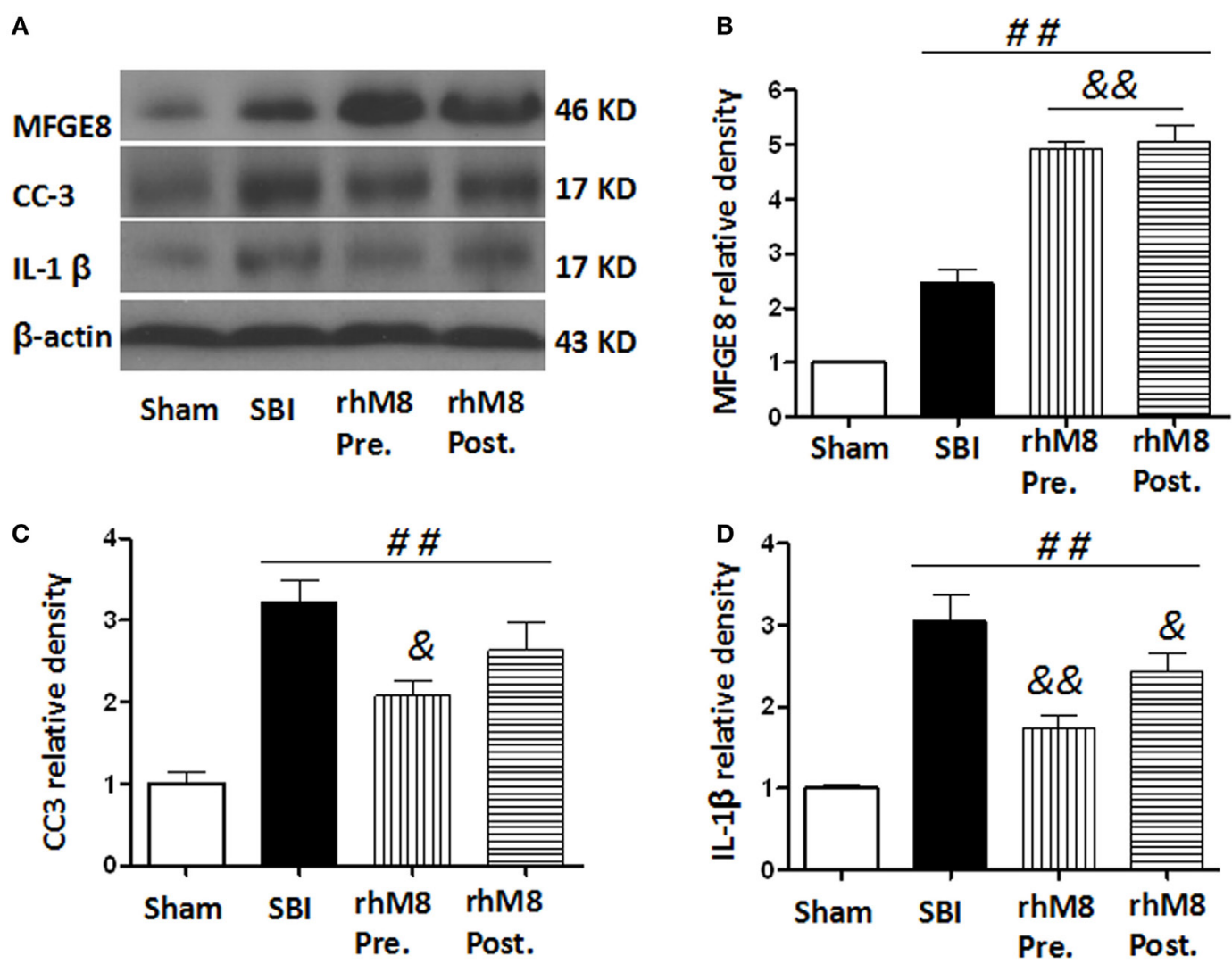

FIGURE 6 | The different effects between recombinant milk fat globule-EGF factor-8 (rhMFGE8) pretreatment (rhM8-pre) and rhMFGE8 posttreatment (rhM8-post) on anti-apoptosis and anti-inflammation at $24 \mathrm{~h}$ after surgical brain injury (SBI). Representative blots (A) and quantitative analysis of the relative densities of MFGE8 (B), cleaved caspase-3 (CC3) (C), and interleukine-1 beta (IL-1 $\beta$ ) (D) indicated that the expressions of MFGE8, CC3, and IL-1 $\beta$ were significantly increased at $24 \mathrm{~h}$ after SBI. Both rhM8-pre and rhM8-post significantly increased the expression of MFGE8. Meanwhile, only rhM8-pre significantly decreased the level of CC3, but not rhM8-post. In addition, both rhM8-pre and rhM8-post significantly decreased the level of IL-1 $\beta$, but rhM8-pre had better inhibiting effect. $n=6$ for each group. ${ }^{\# \# P}<0.01$ vs. sham group, ${ }^{\&} P<0.05$ vs. SBI group, and ${ }^{\& \&} P<0.01$ vs. SBI group.

Inflammatory mediators likely play a key role in the traumatic (cortical incision) and ischemic (brain retraction) injury associated with SBI (2-6), similar to other brain injuries (7-9). As a vital process to prevent inflammation, apoptotic cells need to be rapidly and efficiently cleared $(26,27)$. If apoptotic cells are not cleared by phagocytosis, they will eventually undergo secondary necrosis and stimulate an inflammatory response $(30,31)$. MFGE8 can promote phagocytosis and inhibit inflammation (16). Our data showed that the expression of CC3 was significantly increased $3 \mathrm{~h}$ after SBI, but the increase in MFGE8 protein level was not significant. Similar to the SAH model (16), endogenous MFGE8 protein expression began to increase early after the injury, which was not sufficient, and thus, phagocytosis was limited. Only a portion of the phagocytic cells were cleared, and most apoptotic cells showed secondary necrosis, in which the release of dangerous contents led to increased IL- $1 \beta$ protein level, as shown in our results. The potential damage can be alleviated by exogenous MFGE8, which mediated the removal of apoptotic bodies via its affinity to phosphatidylserine and decreased inflammation. In the present study, pretreatment with rhMFGE8, which remedied the shortage of exogenous
MFGE8, significantly decreased IL-1 protein level and brain water content and significantly alleviated neurological deficits in the SBI model. If administration of rhMFGE8 occurred after the injury, the apoptotic cells were not cleared rapidly, and secondary necrosis and inflammatory responses were observed. Meanwhile, endogenous MFGE8 protein was increased at later time periods, and exogenous MFGE8 was not as important as it was before the injury. Thus, rhMFGE8 posttreatment had poor therapeutic efficacy in the present study. The time point of administering rhMFGE8 influenced its effects, which may explain the dual effects of MFGE8 in different studies.

In addition, literature reports have shown that MFGE8 has anti-inflammatory activity through the activated $\mathrm{Nrf} / \mathrm{HO}-1$ pathway (16) and inhibiting neutrophil migration via integrinav $\beta 3$-dependent MAP kinase activation (32). Meanwhile, the deceased inflammation, which was inhibited predominantly by clearance of apoptotic cells, had anti-apoptosis effects (11,33-35).

However, in real patients, the outcome after craniotomy is usually assessed weeks to months later. There might be less apoptosis initially when using MFGE8, but eventually all groups (treated and non-treated) might have similar neurological 

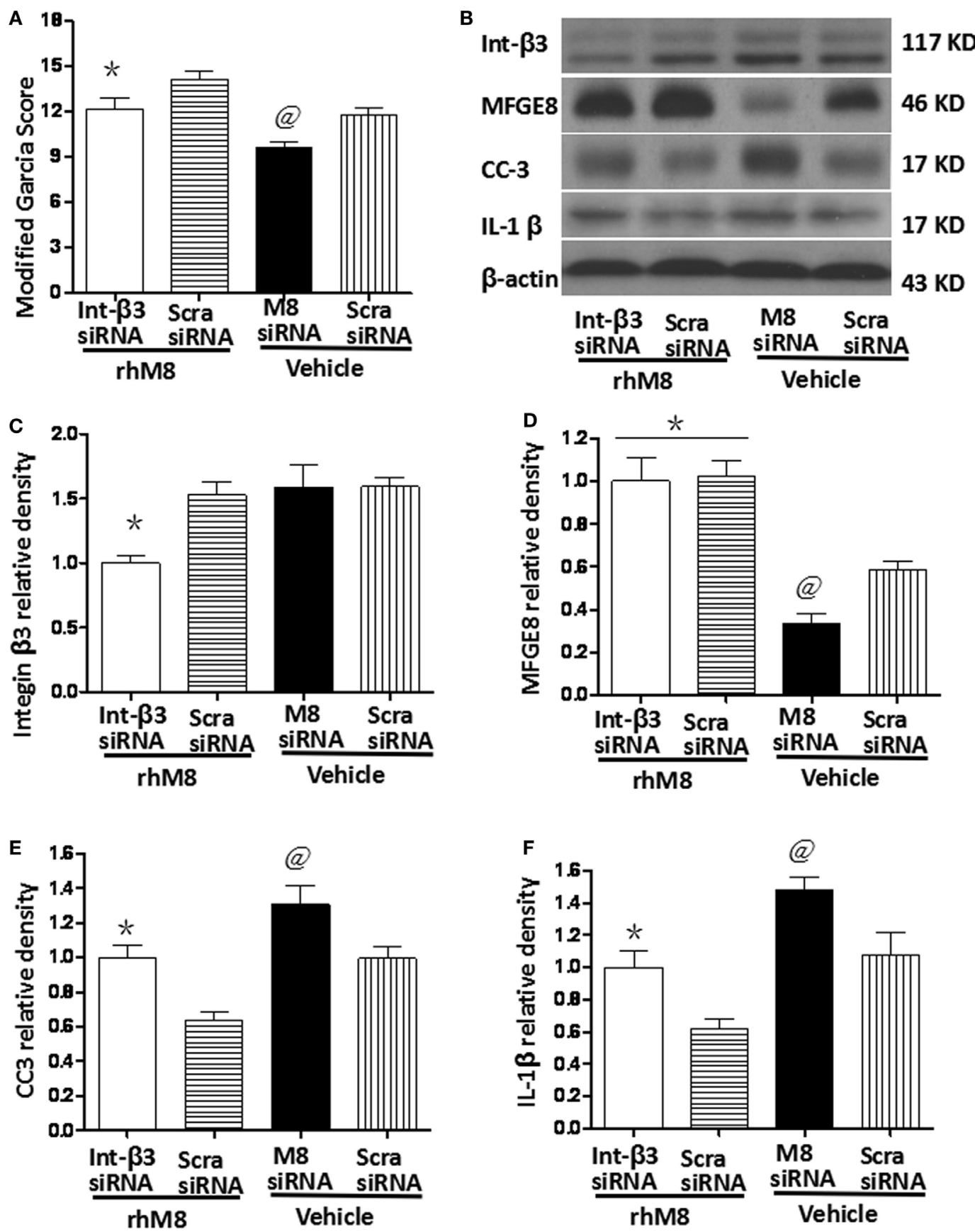

FIGURE 7 | Knockdown endogenous milk fat globule-EGF factor-8 (MFGE8) increased cleaved caspase-3 (CC3) and IL-1 expression. Knockdown integrin- $\beta 3$ receptor abolished the neuroprotective effects of recombinant milk fat globule-EGF factor-8 (rhMFGE8) treatment at $24 \mathrm{~h}$ after surgical brain injury (SBI). (A) MFGE8 siRNA significantly deteriorated the modified Garcia score at $24 \mathrm{~h}$ after SBI, and integrin- $\beta 3$ siRNA offset the ameliorating neurological function of rhMFGE8. Representative blots (B) and quantitative analysis of integrin $\beta 3$, MFGE8, CC3, and interleukine-1 beta (IL-1 $\beta$ ) (C-F) show MFGE8 siRNA significantly decreased MFGE8 expression (D) and increased CC3 and IL-1 expression (E,F), and the level of integrin $\beta 3$ was significantly decreased by integrin- $\beta 3$ siRNA (C) which abolished the effect of MFGE8 decreasing the level of CC3 and IL-1 (E,F). $n=6$ for each group. ${ }^{*} P<0.05$ vs. scramble siRNA + rhMFGE8 (scram siRNA + rhMFGE8) group and ${ }^{\circledR} P<0.05$ vs. scramble siRNA + vehical (scram siRNA + vehical) group.

outcomes. Therefore, the long-term assessment after MFGE8 treatment should be evaluated in the further study. In addition, if the craniotomy is being performed to treat cancer, MFGE8 (which decreases apoptosis) might not be favorable. Thus,
MFGE8 pretreatment may only suitable for the craniotomy for the patients except brain tumor excision, but further experiments should be performed to observe the effects of MFGE8 for the brain tumor cells. 
In the current study, we found that rhMFGE8 pretreatment effectively alleviated neurological deficits and decreased brain water content and apoptotic cells in the SBI model through the MFGE8/integrin- $\beta 3$ pathway, and treatment time was an important factor in achieving curative effects. Clinical, stroke and trauma patients cannot be cured with pretreatment, but SBI patients can. MFGE8 pretreatment may serve as a promising therapeutic strategy for SBI patients.

\section{ETHICS STATEMENT}

All experimental protocols of this study were approved by the Ethics Committee of Central South University and performed according to the eighth edition of the National Institutes of Health Guide for the Care and Use of Laboratory Animals.

\section{REFERENCES}

1. Manninen PH, Raman SK, Boyle K, El-Beheiry H. Early postoperative complications following neurosurgical procedures. Can J Anaesth (1999) 46:7-14. doi:10.1007/BF03012507

2. Matchett G, Hahn J, Obenaus A, Zhang J. Surgically induced brain injury in rats: the effect of erythropoietin. J Neurosci Methods (2006) 158:234-41. doi:10.1016/j.jneumeth.2006.06.003

3. Jadhav V, Zhang JH. Surgical brain injury: prevention is better than cure. Front Biosci (2008) 13:3793-7. doi:10.2741/2968

4. Manaenko A, Sun X, Kim CH, Yan J, Ma Q, Zhang JH. PAR-1 antagonist SCH79797 ameliorates apoptosis following surgical brain injury through inhibition of ASK1-JNK in rats. Neurobiol Dis (2013) 50:13-20. doi:10.1016/j. nbd.2012.09.004

5. Huang L, Sherchan P, Wang Y, Reis C, Applegate RL II, Tang J, et al. Phosphoinositide 3-kinase gamma contributes to neuroinflammation in a rat model of surgical brain injury. J Neurosci (2015) 35:10390-401. doi:10.1523/ JNEUROSCI.0546-15.2015

6. Sherchan P, Huang L, Wang Y, Akyol O, Tang J, Zhang JH. Recombinant Slit2 attenuates neuroinflammation after surgical brain injury by inhibiting peripheral immune cell infiltration via Robo1-srGAP1 pathway in a rat model. Neurobiol Dis (2016) 85:164-73. doi:10.1016/j.nbd.2015.11.003

7. Esiri MM. The interplay between inflammation and neurodegeneration in CNS disease. J Neuroimmunol (2007) 184:4-16. doi:10.1016/j.jneuroim. 2006.11.013

8. Wang Q, Tang XN, Yenari MA. The inflammatory response in stroke. J Neuroimmunol (2007) 184:53-68. doi:10.1016/j.jneuroim.2006.11.014

9. Williams AJ, Wei HH, Dave JR, Tortella FC. Acute and delayed neuroinflammatory response following experimental penetrating ballistic brain injury in the rat. J Neuroinflammation (2007) 4:17. doi:10.1186/1742-2094-4-17

10. Fink SL, Cookson BT. Apoptosis, pyroptosis, and necrosis: mechanistic description of dead and dying eukaryotic cells. Infect Immun (2005) 73:1907-16. doi:10.1128/IAI.73.4.1907-1916.2005

11. Zhang F, Shah KG, Qi L, Wu R, Barrera R, Nicastro J, et al. Milk fat globule epidermal growth factor-8 mitigates inflammation and tissue injury after hemorrhagic shock in experimental animals. J Trauma Acute Care Surg (2012) 72:861-9. doi:10.1097/TA.0b013e318249a97c

12. Xi G, Strahle J, Hua Y, Keep RF. Progress in translational research on intracerebral hemorrhage: is there an end in sight? Prog Neurobiol (2014) 115:45-63. doi:10.1016/j.pneurobio.2013.09.007

13. Oshima K, Aoki N, Negi M, Kishi M, Kitajima K, Matsuda T. Lactationdependent expression of an mRNA splice variant with an exon for a multiply O-glycosylated domain of mouse milk fat globule glycoprotein MFG-E8. Biochem Biophys Res Commun (1999) 254:522-8. doi:10.1006/bbrc. 1998.0107

14. Akakura S, Singh S, Spataro M, Akakura R, Kim JI, Albert ML, et al. The opsonin MFG-E8 is a ligand for the alphavbeta5 integrin and triggers DOCK180-dependent Racl activation for the phagocytosis of apoptotic cells. Exp Cell Res (2004) 292:403-16. doi:10.1016/j.yexcr.2003.09.011

\section{AUTHOR CONTRIBUTIONS}

FL, HF, and JZ conceived and designed the experiments. YX, GL, YZ, KR, and TH performed the experiments. YX, GL, and FL analyzed the data. FL and YC wrote and revised the manuscript. FL gave the final approval for the manuscript to be published.

\section{FUNDING}

This work was supported by the New Xiangya Talent Project of the Third Xiangya Hospital of Central South University (No. 20160302 to FL). The funders had no role in study design, data collection and analysis, decision to publish, or preparation of the manuscript.

15. Takahashi K, Rochford CD, Neumann H. Clearance of apoptotic neurons without inflammation by microglial triggering receptor expressed on myeloid cells-2. J Exp Med (2005) 201:647-57. doi:10.1084/jem.20041611

16. Liu F, Hu Q, Li B, Manaenko A, Chen Y, Tang J, et al. Recombinant milk fat globule-EGF factor-8 reduces oxidative stress via integrin beta3/nuclear factor erythroid 2-related factor 2/heme oxygenase pathway in subarachnoid hemorrhage rats. Stroke (2014) 45:3691-7. doi:10.1161/STROKEAHA.114.006635

17. Cheyuo C, Jacob A, Wu R, Zhou M, Qi L, Dong W, et al. Recombinant human MFG-E8 attenuates cerebral ischemic injury: its role in anti-inflammation and anti-apoptosis. Neuropharmacology (2012) 62:890-900. doi:10.1016/j. neuropharm.2011.09.018

18. Deroide N, Li X, Lerouet D, Van Vre E, Baker L, Harrison J, et al. MFGE8 inhibits inflammasome-induced IL-1beta production and limits postischemic cerebral injury. J Clin Invest (2013) 123:1176-81. doi:10.1172/JCI65167

19. Boddaert J, Kinugawa K, Lambert JC, Boukhtouche F, Zoll J, Merval R, et al. Evidence of a role for lactadherin in Alzheimer's disease. Am J Pathol (2007) 170:921-9. doi:10.2353/ajpath.2007.060664

20. Li E, Noda M, Doi Y, Parajuli B, Kawanokuchi J, Sonobe Y, et al. The neuroprotective effects of milk fat globule-EGF factor 8 against oligomeric amyloid beta toxicity. J Neuroinflammation (2012) 9:148. doi:10.1186/1742-2094-9-148

21. Kranich J, Krautler NJ, Falsig J, Ballmer B, Li S, Hutter G, et al. Engulfment of cerebral apoptotic bodies controls the course of prion disease in a mouse strain-dependent manner. J Exp Med (2010) 207:2271-81. doi:10.1084/ jem.20092401

22. Kinugawa K, Monnet Y, Lu L, Bekaert AJ, Thery C, Mallat Z, et al. MFGE8 does not orchestrate clearance of apoptotic neurons in a mouse model of Parkinson's disease. Neurobiol Dis (2013) 51:192-201. doi:10.1016/j.nbd.2012.11.010

23. Neher JJ, Emmrich JV, Fricker M, Mander PK, Thery C, Brown GC. Phagocytosis executes delayed neuronal death after focal brain ischemia. Proc Natl Acad Sci U S A (2013) 110:E4098-107. doi:10.1073/pnas.1308679110

24. Liu F, Chen Y, Hu Q, Li B, Tang J, He Y, et al. MFGE8/Integrin beta3 pathway alleviates apoptosis and inflammation in early brain injury after subarachnoid hemorrhage in rats. Exp Neurol (2015) 272:120-7. doi:10.1016/j.expneurol. 2015.04.016

25. Yan H, Chen Y, Li L, Jiang J, Wu G, Zuo Y, et al. Decorin alleviated chronic hydrocephalus via inhibiting TGF-beta1/Smad/CTGF pathway after subarachnoid hemorrhage in rats. Brain Res (2016) 1630:241-53. doi:10.1016/j. brainres.2015.11.004

26. Savill J, Gregory C, Haslett C. Cell biology. Eat me or die. Science (2003) 302:1516-7. doi:10.1126/science.1092533

27. Hanayama R, Tanaka M, Miyasaka K, Aozasa K, Koike M, Uchiyama Y, et al. Autoimmune disease and impaired uptake of apoptotic cells in MFG-E8deficient mice. Science (2004) 304:1147-50. doi:10.1126/science.1094359

28. Yang C, Hayashida T, Forster N, Li C, Shen D, Maheswaran S, et al. The integrin alpha(v)beta(3-5) ligand MFG-E8 is a p63/p73 target gene in triple-negative breast cancers but exhibits suppressive functions in $\mathrm{ER}(+)$ and erbB2(+) breast cancers. Cancer Res (2011) 71:937-45. doi:10.1158/0008-5472.CAN-10-1471

29. Gagen D, Filla MS, Clark R, Liton P, Peters DM. Activated alphavbeta 3 integrin regulates alphavbeta5 integrin-mediated phagocytosis in trabecular 
meshwork cells. Invest Ophthalmol Vis Sci (2013) 54:5000-11. doi:10.1167/ iovs.13-12084

30. Poon IK, Lucas CD, Rossi AG, Ravichandran KS. Apoptotic cell clearance: basic biology and therapeutic potential. Nat Rev Immunol (2014) 14:166-80. doi:10.1038/nri3607

31. Seifert HA, Pennypacker KR. Molecular and cellular immune responses to ischemic brain injury. Transl Stroke Res (2014) 5:543-53. doi:10.1007/s12975-014-0349-7

32. Aziz M, Yang WL, Corbo LM, Chaung WW, Matsuo S, Wang P. MFG-E8 inhibits neutrophil migration through alphavbeta(3)-integrin-dependent MAP kinase activation. Int J Mol Med (2015) 36:18-28. doi:10.3892/ijmm.2015.2196

33. Rossi AG. L13. Apoptosis, apoptotic cell clearance and resolution of inflammation. Presse Med (2013) 42:536-7. doi:10.1016/j.lpm.2013.01.012

34. Greenlee-Wacker MC. Clearance of apoptotic neutrophils and resolution of inflammation. Immunol Rev (2016) 273:357-70. doi:10.1111/imr.12453
35. Martin M, Blom AM. Complement in removal of the dead - balancing inflammation. Immunol Rev (2016) 274:218-32. doi:10.1111/imr.12462

Conflict of Interest Statement: The authors declare that the research was conducted in the absence of any commercial or financial relationships that could be construed as a potential conflict of interest.

Copyright (c) 2018 Xiao, Li, Chen, Zuo, Rashid, He, Feng, Zhang and Liu. This is an open-access article distributed under the terms of the Creative Commons Attribution License (CC BY). The use, distribution or reproduction in other forums is permitted, provided the original author(s) and the copyright owner are credited and that the original publication in this journal is cited, in accordance with accepted academic practice. No use, distribution or reproduction is permitted which does not comply with these terms. 